\title{
Spatially Homogeneous String Cosmologies
}

\author{
John D. Barrow and Kerstin E. Kunze \\ Astronomy Centre \\ University of Sussex \\ Brighton BN1 9QH \\ U.K.
}

April 10, 2018

\begin{abstract}
We determine the most general form of the antisymmetric $H$-field tensor derived from a purely time-dependent potential that is admitted by all possible spatially homogeneous cosmological models in 3+1-dimensional low-energy bosonic string theory. The maximum number of components of the $H$ field that are left arbitrary is found for each homogeneous cosmology defined by the Bianchi group classification. The relative generality of these string cosmologies is found by counting the number of independent pieces of Cauchy data needed to specify the general solution of Einstein's equations. The hierarchy of generality differs significantly from that characteristic of vacuum and perfect-fluid cosmologies. The degree of generality of homogeneous string cosmologies is compared to that of the generic inhomogenous solutions of the string field equations.
\end{abstract}

PACS numbers: 9880C, 1125, 0420J, 0450

\section{INTRODUCTION}

The low-energy effective action of the bosonic sector of string theory provides cosmological models that might be applicable just below the Planck (or string) energy scale in the very early universe [1]. A number of studies have been made of these cosmologies in order to ascertain the behaviour of simple isotropic and anisotropic universes, investigate the implications of duality, and search for inflationary solutions [2-6]. Many of the traditional questions of general relativistic cosmology can be asked of the cosmological models defined by string theory: do they possess space-time singularities?, what is the generic behaviour of the solutions at late and early times?, what exact solutions can be found in closed form?, and what relation do particular exact solutions have to the general cosmological solution? Since this theory is to be applied at times very close to the Planck epoch it would be unwise to make special assumptions about the form of the 
cosmological solutions. Anisotropies and inhomogeneities could play an important role in the evolution. Indeed, any dimensional reduction process could be viewed as an extreme form of anisotropic evolution in $\mathrm{D}$ dimensions in which three spatial dimensions expand whilst the rest remain static. Because of these irreducible uncertainties about the very early Universe, one would like to understand the general behaviour of wide classes of solution in order to ascertain the relative generality of any particular solution that may be found. A number of studies have focused on obtaining particular solutions for $3+1$ dimensional space-times in cases where spatial homogeneity (and sometimes also isotropy) is assumed for the metric of space-time, where the $H$ field is set to zero [4], or where the $H$ field is included by assuming that it takes a particular form which satisfies its constraints and its equation of motion [5]. For example, Copeland et al, [2], discussed Friedmann and Bianchi type I universes, allowing $* H$ to be time-dependent or space-dependent, respectively. In a second paper, [3], they discussed Bianchi I solutions with a homogeneous antisymmetric tensor field. In [6] (see also [5]) Batakis presented an overview of all possible configurations of a (spatially) homogeneous $H$-field in diagonal Bianchi models with a metric

$$
d s^{2}=-d t^{2}+a_{1}(t)^{2}\left(\omega^{1}\right)^{2}+a_{2}(t)^{2}\left(\omega^{2}\right)^{2}+a_{3}(t)^{2}\left(\omega^{3}\right)^{2}
$$

where $\left\{d t, \omega^{\alpha}\right\}$ is the standard basis. However, in this paper the Bianchi models are not assumed to be diagonal.

The form of the $H$-field derived from a time-dependent potential will be determined in all four-dimensional space-times with homogeneous three-spaces. These three-spaces were first classified by Bianchi [7] and have been extensively studied in the cosmological context following their introduction into cosmology by Taub [8]. They provide us with the general class of cosmological models whose solutions are determined by ordinary differential equations in time. By generalising a procedure used to study electromagnetic fields in spatially homogeneous cosmological models by Hughston and Jacobs [10], we can determine the maximum number of components permitted for the $H$ field in each of the Bianchi cosmologies. This enables us to determine the number of degrees of freedom which define the string cosmology of each case. The results are interesting. The Bianchi types containing the most general geometries place the most restrictions upon the presence of the $H$ field.

The string world sheet action for a closed bosonic string in a background field including all the massless states of the string as part of the background is given by, [1],

$$
S=-\frac{1}{4 \pi \alpha^{\prime}} \int d^{2} \sigma\left\{\sqrt{h} h^{\alpha \beta} \partial_{\alpha} X^{\mu} \partial_{\beta} X^{\nu} g_{\mu \nu}\left(X^{\rho}\right)+\epsilon^{\alpha \beta} \partial_{\alpha} X^{\mu} \partial_{\beta} X^{\nu} B_{\mu \nu}\left(X^{\rho}\right)+\alpha^{\prime} \sqrt{h} \phi\left(X^{\rho}\right) R^{(2)}\right\}
$$

where $h^{\alpha \beta}$ is the 2-dimensional worldsheet metric, $R^{(2)}$ the worldsheet Ricci scalar, $\epsilon^{\alpha \beta}$ the worldsheet antisymmetric tensor, $B_{\mu \nu}\left(X^{\rho}\right)$ the antisymmetric tensor field, $g_{\mu \nu}\left(X^{\rho}\right)$ the background space-time metric (graviton), $\phi\left(X^{\rho}\right)$ the dilaton, $\alpha^{\prime}$ is the inverse string tension, and the functions $X^{\rho}(\sigma)$ map the string worldsheet into the physical D-dimensional space-time manifold. 
For the consistency of string theory it is essential that local scale invariance holds. Imposing this condition results in equations of motion for the fields $g_{\mu \nu}, B_{\mu \nu}$ and $\phi$ which can be derived to lowest order in $\alpha^{\prime}$ from the low-energy effective action

$$
S=\int d^{D} x \sqrt{-g} e^{-\phi}\left(R+g^{a b} \partial_{a} \phi \partial_{b} \phi-\frac{1}{12} H^{a b c} H_{a b c}-\Lambda\right) .
$$

In this paper we assume a vanishing cosmological constant, $\Lambda$.

In a cosmological context it is generally assumed that by some means all but four of the 10 or 26 dimensions of space-time are compactified, leaving an expanding 3+1-dimensional space-time $(D=4)$. Since we are interested in cosmological solutions of the field equations derived from the variation of this action, we adopt the Einstein frame by making the conformal transformation

$$
g_{a b} \rightarrow e^{-\phi} g_{a b}
$$

In this frame the 4-dimensional string field equations and the equations of motion are given by (indices run $0 \leq a, b, c \leq 3$ and $1 \leq \alpha, \beta \leq 3$ ),

$$
\begin{aligned}
R_{a b}-\frac{1}{2} g_{a b} R & =\kappa^{2}\left({ }^{(\phi)} T_{a b}+{ }^{(H)} T_{a b}\right), \\
\nabla_{a}\left(e^{-2 \phi} H^{a b c}\right) & =0, \\
\square \phi+\frac{1}{6} e^{-2 \phi} H_{a b c} H^{a b c} & =0,
\end{aligned}
$$

where $\kappa^{2}=8 \pi G$ is the 4 -dimensional Einstein gravitational coupling and

$$
\begin{aligned}
{ }^{(\phi)} T_{a b} & \equiv \frac{1}{2}\left(\phi_{, a} \phi_{, b}-\frac{1}{2} g_{a b} \phi_{, c} \phi^{, c}\right), \\
{ }^{(H)} T_{a b} & \equiv \frac{1}{12} e^{-2 \phi}\left(3 H_{a c d} H_{b}{ }^{c d}-\frac{1}{2} g_{a b} H_{m l k} H^{m l k}\right) .
\end{aligned}
$$

The 3-geometries of the nine spatially homogeneous cosmological solutions of these equations are defined by the Bianchi classification of homogeneous spaces (with the exception of the Kantowski-Sachs universe, [9], which has a four-dimensional group of motions but no threedimensional subgroup). In these Bianchi models (e.g. [13]) the spacelike hypersurfaces are invariant under the group $G_{3}$ of isometries whose generators are 3 Killing vectors $\xi_{\alpha}$. These hypersurfaces can be described by an invariant vector basis $\left\{X_{\alpha}\right\}$ satisfying

$$
\mathcal{L}_{\xi_{\beta}} X_{\alpha}=\left[\xi_{\beta}, X_{\alpha}\right]=0
$$

where $\mathcal{L}_{\xi_{\beta}}$ is the Lie derivative in the direction of $\xi_{\beta}$. The timelike direction $X_{0}$ is chosen to be orthogonal to the invariant spacelike hypersurfaces obeying

$$
\mathcal{L}_{\xi_{\beta}} X_{0}=\left[\xi_{\beta}, X_{0}\right]=0
$$


Dual to $\left\{X_{\alpha}\right\}$ is the basis of one-forms $\left\{\omega^{\mu}\right\}$ satisfying

$$
\omega^{\mu}=\frac{1}{2} C_{\kappa \lambda}^{\mu} \omega^{\kappa} \wedge \omega^{\lambda}
$$

Spatial homogeneity is expressed by the following conditions on $\phi, g$ and $H$

$$
\begin{gathered}
\mathcal{L}_{\xi_{\alpha}} \phi=0, \\
\mathcal{L}_{\xi_{\alpha}} g=0, \\
\mathcal{L}_{\xi_{\alpha}} H=0 \Rightarrow \mathcal{L}_{\xi_{\alpha}}(* H)=0 .
\end{gathered}
$$

The definition and properties of the Lie derivative imply that $\mathcal{L}_{\xi_{\alpha}} \phi=\xi_{\alpha} \phi=0$. Expanding $* H$ in the invariant basis (that is, $* H=V^{0} X_{0}+V^{\alpha} X_{\alpha}$ ), and using its properties, implies then $\xi_{\alpha} V^{0}=0$ and $\xi_{\alpha} V^{\beta}=0$. The Killing vectors in the Bianchi models are spacelike and time independent and this then implies that $\phi$ and $H$ are functions of time only in the standard basis $\left\{d t, \omega^{\alpha}\right\}$. Furthermore, the antisymmetric tensor potential $B$ where $H=d B$ will be assumed to be a function of time only.

We would like to know the general algebraic form of the $H$ field with a time-dependent potential $B$ in these models, determine which Bianchi universes are the most general, and discover whether the assumption of spatial homogeneity reduces the number of independent pieces of Cauchy data below the number needed to specify a generic inhomogeneous solution of the field equations (4)-(8). This analysis of the allowed components of the $H$-field is most economically performed using differential forms.

\section{THE ANTISYMMETRIC TENSOR FIELD AS A 2-FORM}

There are three equations determining the antisymmetric tensor field: the definition of its field strength (for a closed bosonic string)

$$
H=d B
$$

which implies the second equation

$$
d H=0,
$$

and there is the equation of motion, (5),

$$
d(* H)-2(d \phi) \wedge(* H)=0 .
$$


Spatially homogeneous models are described by choosing an orthonormal tetrad,

$$
d s^{2}=\eta_{a b} \sigma^{a} \sigma^{b}
$$

where $\eta_{a b}=\operatorname{diag}(-1,1,1,1)$, and specifying the 1 -forms $\sigma^{a}[10,13]$ as

$$
\begin{aligned}
\sigma^{0} & =N(\Omega) d \Omega \\
\sigma^{\alpha} & =e^{-\Omega} b_{\beta}^{\alpha} \omega^{\beta} .
\end{aligned}
$$

Here, the $\omega^{\alpha}$ obey the algebra

$$
d \omega^{\alpha}=\frac{1}{2} C_{\beta \gamma}^{\alpha} \omega^{\beta} \wedge \omega^{\gamma},
$$

where $C_{\beta \gamma}^{\alpha}$ are the structure constants of the possible isometry groups which define the homogeneous 3 -spaces, and the $b_{\beta}^{\alpha}$ are symmetric matrices which depend only on the time coordinate $\Omega$. Since $B$ is a 2 -form, it can be decomposed as

$$
B=B_{0 \alpha} \sigma^{0} \wedge \sigma^{\alpha}+B_{\alpha \beta} \sigma^{\alpha} \wedge \sigma^{\beta}=Q_{0 \kappa} d \Omega \wedge \omega^{\kappa}+S_{\kappa \mu} \omega^{\kappa} \wedge \omega^{\mu}
$$

where

$$
\begin{gathered}
Q_{0 \kappa}(\Omega) \equiv N B_{0 \alpha} e^{-\Omega} b_{\kappa}^{\alpha}, \\
S_{\kappa \mu}(\Omega) \equiv e^{-2 \Omega} B_{\alpha \beta} b_{\kappa}^{\alpha} b_{\mu}^{\beta} .
\end{gathered}
$$

Hence, $H=d B$ is given by

$$
H=\left(S_{\alpha \beta \mid \Omega}-\frac{1}{2} C_{\alpha \beta}^{\kappa} Q_{0 \kappa}\right) d \Omega \wedge \omega^{\alpha} \wedge \omega^{\beta}+\frac{1}{2} S_{\kappa \mu} C_{\alpha \beta}^{[\kappa} \omega^{\mu]} \wedge \omega^{\alpha} \wedge \omega^{\beta} .
$$

This expression can be analysed further if we introduce the Ellis-MacCallum [11] decomposition of the structure constants into the matrix $m_{\alpha \beta}$ and the vector $a_{\beta}$,

$$
C_{\alpha \beta}^{\gamma}=\epsilon_{\alpha \beta \mu} m^{\mu \gamma}+\delta_{\beta}^{\gamma} a_{\alpha}-\delta_{\alpha}^{\gamma} a_{\beta},
$$

so (18) becomes

$$
H=\left(S_{\alpha \beta \mid \Omega}-\frac{1}{2} C_{\alpha \beta}^{\kappa} Q_{0 \kappa}\right) d \Omega \wedge \omega^{\alpha} \wedge \omega^{\beta}+2 a_{\alpha} S_{\kappa \mu} \omega^{\mu} \wedge \omega^{\alpha} \wedge \omega^{\kappa} .
$$

The structure constants satisfy a Jacobi identity which leaves $C_{\alpha \beta}^{\gamma}$ with a maximum of 6 independent components. Since the lagrangian is invariant under the gauge transformation 
$B_{a b} \rightarrow B_{a b}+\partial_{[a} \Lambda_{b]}$, we can always choose $\Lambda$ such that $Q_{0 \kappa}=-\partial_{[0} \Lambda_{\kappa]}=-\partial_{0} \Lambda_{\kappa}$, and set $Q_{0 \kappa}$ to be zero.

The nine Bianchi-type universes fall into two classes, A and B, distinguished by whether the constant $a$ is zero or non-zero respectively [11]. From (20) we see that $H$ has no purely spatial components in Class A models.

$H$ is also given by

$$
\begin{aligned}
H & =H_{a b c} \sigma^{a} \wedge \sigma^{b} \wedge \sigma^{c} \\
& =H_{0 \alpha \beta} \sigma^{0} \wedge \sigma^{\alpha} \wedge \sigma^{\beta}+H_{\alpha \beta \gamma} \sigma^{\alpha} \wedge \sigma^{\beta} \wedge \sigma^{\gamma} \\
& =X_{0 \kappa \lambda} d \Omega \wedge \omega^{\kappa} \wedge \omega^{\lambda}+Y_{\kappa \lambda \mu} \omega^{\kappa} \wedge \omega^{\lambda} \wedge \omega^{\mu}
\end{aligned}
$$

where

$$
\begin{aligned}
& X_{0 \kappa \lambda}=X_{0 \kappa \lambda}(\Omega) \equiv N e^{-2 \Omega} b_{\kappa}^{\alpha} b_{\lambda}^{\beta} H_{0 \alpha \beta}, \\
& Y_{\kappa \lambda \mu}=Y_{\kappa \lambda \mu}(\Omega) \equiv e^{-3 \Omega} b_{\kappa}^{\alpha} b_{\lambda}^{\beta} b_{\mu}^{\gamma} H_{\alpha \beta \gamma} .
\end{aligned}
$$

Therefore $d H=0$ implies

$$
Y_{\kappa \lambda \mu \mid \Omega} d \Omega \wedge \omega^{\kappa} \wedge \omega^{\lambda} \wedge \omega^{\mu}+\frac{1}{2} X_{0 \kappa \lambda}\left(C_{\alpha \beta}^{\kappa} \omega^{\lambda}-C_{\alpha \beta}^{\lambda} \omega^{\kappa}\right) \wedge \omega^{\alpha} \wedge \omega^{\beta} \wedge d \Omega=0 .
$$

Using the expression (19) for the structure constants, and noting that the 3-dimensional Levi-Civita symbol is defined by $\epsilon=\sqrt{\operatorname{det} g_{\alpha \beta}} \omega^{1} \wedge \omega^{2} \wedge \omega^{3}$, eq. (24) becomes

$$
\left(Y_{\kappa \lambda \mu \mid \Omega}-2 X_{0 \mu \kappa} a_{\lambda}\right) d \Omega \wedge \omega^{\kappa} \wedge \omega^{\lambda} \wedge \omega^{\mu}=0 .
$$

The dual, $* \lambda$, of an n-dimensional p-form $\lambda$ is defined by the Levi-Civita symbol as [12]

$$
* \lambda_{b_{1} \ldots b_{n-p}}=\frac{1}{p !} \lambda^{a_{1} \ldots a_{p}} \epsilon_{a_{1} \ldots a_{p} b_{1} \ldots b_{n-p}} .
$$

Hence, $* H$ is a 1 -form given by

$$
* H=\frac{1}{6} H^{b c d} \epsilon_{b c d a} \sigma^{a}=U d \Omega+V_{\alpha} \omega^{\alpha},
$$

where

$$
U \equiv U(\Omega) \equiv \frac{1}{6} H^{\alpha \beta \gamma} \epsilon_{\alpha \beta \gamma 0} N
$$




$$
V_{\alpha} \equiv V_{\alpha}(\Omega) \equiv \frac{1}{6} H^{a b c} \epsilon_{a b c \kappa} b_{\alpha}^{\kappa} e^{-\Omega}
$$

and so

$$
d * H=V_{a \mid \Omega} d \Omega \wedge \omega^{\alpha}+\frac{1}{2} V_{\alpha} C_{\beta \gamma}^{\alpha} \omega^{\beta} \wedge \omega^{\gamma} .
$$

Since $\phi=\phi(\Omega)$, we have $d \phi=\phi_{\mid \Omega} d \Omega$ and equation (11) reads

$$
\left(V_{\alpha \mid \Omega}-2 \phi_{\mid \Omega} V_{\alpha}\right) d \Omega \wedge \omega^{\alpha}+\frac{1}{2} V_{\alpha} C_{\beta \gamma}^{\alpha} \omega^{\beta} \wedge \omega^{\gamma}=0
$$

hence,

$$
\begin{aligned}
V_{\alpha \mid \Omega}-2 \phi_{\mid \Omega} V_{\alpha} & =0 \\
\frac{1}{2} V_{\alpha} C_{[\beta \gamma]}^{\alpha} & =0 .
\end{aligned}
$$

Notice that the constraint (32) is preserved in time. Contracting (31) with $C_{[\beta \gamma]}^{\alpha}$ gives $\left(V_{\alpha} C_{[\beta \gamma]}^{\alpha}\right)_{\mid \Omega}=0$ so that if (32) is satisfied at one time it holds at all times. Eqn. (32) implies

$$
\epsilon^{\beta \gamma \delta} V_{\alpha} C_{\beta \gamma}^{\alpha}=0
$$

which can be rewritten as

$$
V_{\alpha}\left(m^{\delta \alpha}+a_{\beta} \epsilon^{\beta \alpha \delta}\right)=0,
$$

and so, by (20), we have

$$
H=X_{0 \kappa \lambda} d \Omega \wedge \omega^{\kappa} \wedge \omega^{\lambda}+Y_{\kappa \lambda \mu} \omega^{\mu} \wedge \omega^{\alpha} \wedge \omega^{\kappa},
$$

with

$$
X_{0 \kappa \lambda}=S_{[\kappa \lambda] \mid \Omega}-\frac{1}{2} C_{[\kappa \lambda]}^{\nu} Q_{0 \nu} \text { and } Y_{\kappa \lambda \mu}=2 a_{[\alpha} S_{\kappa \mu]} .
$$

Eqn. (25) implies

$$
C_{[\mu \kappa}^{\alpha} a_{\lambda]} Q_{0 \alpha}=0
$$

and (31) can be integrated to give

$$
V_{\alpha}=e^{2 \phi} K_{\alpha}
$$


where $K_{\alpha}$ is a constant spatial 3 -vector of integration.

Since $*(* H)=H$, we have

$$
\begin{aligned}
X^{0 \alpha \beta} & =-\epsilon^{0 \alpha \beta \gamma} V_{\gamma} \\
& =\epsilon^{0 \alpha \beta \gamma} e^{2 \phi} K_{\gamma},
\end{aligned}
$$

where the minus sign has been absorbed into the constant spatial 3-vector $K_{\gamma}$.

\section{TABLE 1}

Table 1 displays the restrictions on the spatial components of $* H$ imposed by the constraint equation (34) for the different Bianchi types [11,13], together with the components of the homogeneous antisymmetric tensor field strength $H$ in the standard basis $\left\{d \Omega, \omega^{\alpha}\right\}$ which are given by eqn. (20). Note that in Class A, eqn. (23) implies $Y_{123}=0$, and the contravariant components of $Y_{\alpha \beta \gamma}$ are obtained by raising the indices using $g_{a b}$ given by

$$
\begin{gathered}
g_{00}=-N^{2}(\Omega) \\
g_{\alpha \beta}=e^{-2 \Omega} \sum_{\gamma} b_{\alpha}^{\gamma} b_{\beta}^{\gamma} .
\end{gathered}
$$

In Class B, eqn.(23) implies that $Y_{123}=2 a_{[2} S_{31]}=2 a_{3} S_{12}$. The matrix $\alpha$ which specifies the Ellis-MacCallum symbol $\mathbf{m}=m_{\alpha \beta}$ is defined by, [11], the matrix

$$
\alpha=\left(\begin{array}{lll}
0 & 1 & 0 \\
1 & 0 & 0 \\
0 & 0 & 0
\end{array}\right)
$$

\section{COUNTING DEGREES OF FREEDOM}

Consider first the question of how many independently arbitrary spatial functions are required to specify generic initial data for the system of string field equations (4)-(8). In a synchronous frame we require $6 g_{\alpha \beta}, 6 \dot{g}_{\alpha \beta}, 3$ components of the $H$ field, together with values of $\phi$ and $\dot{\phi}$. This amounts to 17 functions, but we can remove 4 by using the coordinate covariance of the theory, another 4 by using the $R_{0 a}$ constraint equations, and another 1 by using the $\phi$ equation, (6). This leaves 8 independent functions of three spatial variables to specify a general solution of the field equations (4)-(8). If special symmetries are assumed for the solutions of the field equations then some of the metric components and their time derivatives may be absent but some of the algebraic $R_{0 a}$ constraints may be identically satisfied. As a result, the number 
of functions characterising the most general solution compatible with some symmetry may be specified by fewer functions (or by lower-dimensional functions) than the general solution.

Spatially homogeneous cosmological models will be determined by some number of independently arbitrary constants rather than spatial functions. If spatially homogeneous string cosmologies are representative of the most general inhomogeneous string cosmologies then it is necessary (although not necessarily sufficient) that they be characterised by 8 independent arbitrary constants. When the $H$ field vanishes in eqns. (4)-(8), so they reduce Einstein's equations for a free scalar field, the number of arbitrary functions is required to characterise the general inhomogeneous solution equals the number of constants required for the general homogeneous solution. This equivalence also holds for Einstein's equations with a perfect fluid (or in vacuum), where 8 (or 4) functions specify a general inhomogeneous solution and 8 (or 4) constants specify Bianchi types $\mathrm{VI}_{h}, \mathrm{VII}_{h}, \mathrm{VIII}$, and IX, $[15,16]$. We shall now investigate the degree of generality of the different Bianchi type solutions of the string field equations when the $H$ field is present.

In order to determine how many free parameters are allowed in the different Bianchi models, consider the field equations, (4), for spatially homogeneous universes in the standard basis $\left\{d \Omega, \omega^{\alpha}\right\}$. The components of the Ricci tensor are given by [14]

$$
\begin{aligned}
& R_{00}=-\dot{\theta}-\theta_{\alpha \beta} \theta^{\alpha \beta} \\
& R_{0 \alpha}=3 a_{\gamma} \theta_{\alpha}^{\gamma}-a_{\alpha} \theta+\epsilon_{\gamma \alpha \tau} m^{\tau \beta} \theta_{\beta}^{\gamma}, \\
& R_{\alpha \beta}=\dot{\theta}_{\alpha \beta}+\theta \theta_{\alpha \beta}-2 \theta_{\alpha \gamma} \theta_{\beta}^{\gamma}+\Gamma_{\lambda \gamma}^{\gamma} \Gamma_{\alpha \beta}^{\lambda}-\Gamma_{\lambda \beta}^{\gamma} \Gamma_{\alpha \gamma}^{\lambda}+C_{\gamma \beta}^{\kappa} \Gamma_{\alpha \kappa}^{\gamma},
\end{aligned}
$$

where $\theta_{\alpha \beta}=\frac{1}{2} g_{\alpha \beta \mid \Omega}, \theta \equiv \theta_{\alpha}^{\alpha}$ and the Ellis-MacCallum parametrization, (19), has been used to express the spatial curvature terms in (44) and (45).

The string field equations give 10 equations for the 6 components of the symmetric metric $g_{\alpha \beta}$, so there are at most 4 constraint equations. The initial data for $g_{\alpha \beta}$ consist of 12 independent constants: $6 g_{\alpha \beta}$ and $6 \dot{g}_{\alpha \beta}$. These are reduced by $(9-p+1)$ due to the fact that there are $9-p+1$ parameters of triad freedom to put the group structure constants into their canonical Ellis-MacCallum form [14]. The parameter $p$ is the number of independent group structure constants and $0 \leq p \leq 6$. Their values are given below, and in Table 1, for each Bianchi group type. The number of independent constants is reduced by a further $4-r$ due to the constraint equations, where $r$ counts the number of field equations satisfied identically. Hence, a total of $12-(9-p+1)-(4-r)=p+r-2$ independent constants specify the general solution to equations (4)-(8) for spatially homogeneous universes. To calculate $r$ we must check if any of the field equations are identically satisfied due to a particular choice of the group structure parameters $a_{\beta}$ and $m_{\alpha \beta}$. From eqn. (7) it is clear that the dilaton's contribution to the $R_{0 \alpha}$ equations vanishes identically. The contribution by the $H$ field is determined by $H_{0 c d} H_{\alpha}{ }^{c d}$, but we know from (35)-(36) that $H_{0 c d} H_{\alpha}{ }^{c d}=-X^{0 \beta \gamma} Y_{\alpha \beta \gamma}$, hence $R_{0 \alpha}=0$ for all Class A models.

The equations of motion for $H$ and the constraints they impose have been discussed in section II; Table 1 gives the number of free parameters, $3-u$, to specify initial data for $H$ for each 
group type. The initial conditions for the dilaton $\phi$ require 2 further independent constants: $\phi$ and $\dot{\phi}$, while eqn.(6) determines the dynamics of $\phi$. Therefore the general spatially homogeneous solution(s) to equations (4)-(8) contain

$$
\mathcal{N} \equiv 3+p+r-u
$$

independent arbitrary constants. Using the constraint equations (34) and (44) we can evaluate $p, r, u$, and $\mathcal{N}$ (Bianchi type) explicitly as follows (the values of these parameters are summarised in Table 1).

\section{A. Class A models}

Bianchi I: $R_{0 \alpha}=0$, hence $r=3, p=0, u=0$ and $\mathcal{N}(I)=6$

Bianchi II: $R_{01}=0, R_{02}=-\theta_{1}^{3}, R_{03}=\theta_{1}^{2}$, hence $r=1, p=3, u=1$, and $\mathcal{N}(I I)=6$

Bianchi $V I_{-1}: R_{01}=-\theta_{1}^{3}, R_{02}=\theta_{2}^{3}, R_{03}=\theta_{1}^{1}-\theta_{2}^{2}$, hence $r=0, p=5, u=2$, and $\mathcal{N}\left(V I_{-1}\right)=6$

Bianchi $V I I_{0}: R_{01}=-\theta_{2}^{3}, R_{02}=\theta_{1}^{3}, R_{03}=\theta_{2}^{1}-\theta_{1}^{2}$, hence $r=0, p=5, u=2$, and $\mathcal{N}\left(V I I_{0}\right)=6$

Bianchi VIII: $R_{01}=\theta_{2}^{3}-\theta_{3}^{2}, R_{02}=\theta_{1}^{3}+\theta_{3}^{1}, R_{03}=-\theta_{1}^{2}-\theta_{2}^{1}$, hence $r=0, p=6, u=3$ and $\mathcal{N}(V I I I)=6$

Bianchi IX: $R_{01}=\theta_{2}^{3}-\theta_{3}^{2}, R_{02}=\theta_{3}^{1}-\theta_{1}^{3}, R_{03}=\theta_{1}^{2}-\theta_{2}^{1}$, hence $r=0, p=6, u=3$, and $\mathcal{N}(I X)=6$.

Hence, all Class A models are equally general according to the parameter-counting criterion.

\section{B. Class B models}

Bianchi III: $R_{01}=-2 \theta_{1}^{3}, R_{02}=-\theta_{2}^{3}, R_{03}=\theta_{1}^{1}-\theta_{3}^{3}$, hence $r=0, p=5, u=0$, and $\mathcal{N}(I I I)=8$

Bianchi $I V: R_{01}=-3 \theta_{1}^{3}, R_{02}=-3 \theta_{2}^{3}-\theta_{1}^{3}, R_{03}=-\theta_{3}^{3}+\theta+\theta_{1}^{2}$, hence $r=0, p=5, u=1$, and $\mathcal{N}(I V)=7$

Bianchi $V: R_{01}=-3 \theta_{1}^{3}, R_{02}=-3 \theta_{2}^{3}, R_{03}=-\theta-3 \theta_{3}^{3}$, hence $r=0, p=3, u=1$, and $\mathcal{N}(V)=5$

Bianchi $V I_{h \neq-1}: R_{01}=-(h+2) \theta_{1}^{3}, R_{02}=-(2 h+1) \theta_{2}^{3}, R_{03}=\theta_{1}^{1}+h \theta_{2}^{2}-(h+1) \theta_{3}^{3}$. For special choices of $h$, either $R_{01}$ or $R_{02}$ can be made to vanish identically. The two choices are either $h=-2$ or $h=-\frac{1}{2}$, [15], so that $r(h=-2)=r(h=-1 / 2)=1$. Therefore, we have three cases,

(i) $h=0: r=0, p=5, u=0$, and $\mathcal{N}\left(V I_{0}\right)=8$,

(ii) $h \neq 0, h \neq-2$ and $h \neq-\frac{1}{2}: r=0, p=5, u=1$, and $\mathcal{N}\left(V I_{h}\right)=7$,

(iii) $h=-2$ or $h=-\frac{1}{2}: r=1, p=5, u=1$, and $\mathcal{N}\left(V I_{h \neq-2,-\frac{1}{2}}\right)=8$. 
Bianchi $V I I_{h \neq 0}: R_{01}=-h \theta_{1}^{3}-\theta_{2}^{3}, R_{02}=\theta_{1}^{3}-2 h \theta_{2}^{3}, R_{03}=\theta_{2}^{1}-\theta_{1}^{2}+h \theta_{2}^{2}-h \theta_{3}^{3}$. Since $h$ is a real parameter there are no exceptional cases; hence $r=0, p=5, u=1$, and $\mathcal{N}\left(V I I_{h}\right)=7$.

This analysis of Class B models indicates that they are described by more free parameters than those of Class A and the different Class B models are not equally general like those of Class A. Thus the most general spatially homogeneous solutions of the string equations (4)(8) are those of Bianchi types III and $\mathrm{VI}_{h=0,-\frac{1}{2},-2}$. These cosmological models contain the maximum number of 8 free parameters. These results can be compared with the study of homogeneous pure magnetic or pure electric fields in general-relativistic Bianchi universes carried out by Hughston and Jacobs [10] and Ruban [17] where a similar phenomenon occurs. For a homogeneous magnetic field the most general solution is found to be Bianchi type III. Contrary to what was found for the antisymmetric tensor field strength, the exceptional purely magnetic universes of Bianchi type VI do not contain as many free parameters as the Bianchi III universe. This might be related to the fact that $H$ is a 3 -form and the homogeneous space is three dimensional, which implies that $d H$ does not provide additional constraints on the purely spatial components of $H$, whereas in the case of a Maxwell 2-form, $f$, the differential, $d f$, does give additional constraints on the purely spatial components involving the group structure constants via the Maxwell equations $d f=0=d * f$. However, the number of free parameters in Bianchi models in the cases of the homogeneous pure magnetic fields and homogeneous antisymmetric tensor field strength possess common features. In both cases the generality of the solutions is

the same in Bianchi types IV, $\mathrm{VI}_{h \neq 0,-\frac{1}{2},-2}$ and $\mathrm{VII}_{h \neq 0}$, and the least general model is Bianchi type $\mathrm{V}$.

The hierarchy of generality in the string cosmologies has several interesting features when compared with the situation of vacuum and perfect-fluid universes in general relativity. The most general category of 8-parameter models (types III and $\mathrm{VI}_{h=0,-\frac{1}{2},-2}$ ) does not contain closed universes (ie type IX) as in general relativity, nor does it contain any types which contain isotropic universes as particular cases (ie types $\mathrm{I}, \mathrm{V}, \mathrm{VII}_{0}, \mathrm{VII}_{h}$ or IX). Isotropy is not an open property of homogeneous initial data space. This is related to the fact that the $H$ field is an anisotropic stress: the isotropic limit cannot be obtained with a non-zero $H$ field. This means that the isotropic Friedmann universes appear to be even less representative of the general behaviour of cosmological models in string theory than they are in general relativity. However, a similar situation can arise in general relativity when anisotropic stresses are included.

\section{CONCLUSIONS}

The equations that determine the antisymmetric tensor field in low-energy effective string theory have been investigated in spatially homogeneous Bianchi-type universes. It is found that the homogeneous 3 -form $H$ with a homogeneous potential can have at most three nonvanishing 
components. The number of allowed components were fully classified in Table 1. In Bianchi Class A models the field strength $H$ has no purely spatial components in the standard basis. Bianchi types VIII and IX allow only a time-independent, antisymmetric tensor field, $B_{\mu \nu}$, which implies a vanishing field strength $H$. In the case of Bianchi IX this can be understood in geometrical terms. Each of the Bianchi models corresponds to a group of motions or isometries of spatial hypersurfaces. In the case of Bianchi IX this group is isomorphic to $\mathrm{SO}(3, \mathbb{R})$, which is isomorphic to the three-dimensional rotation group. Since the dual of the antisymmetric tensor field strength, $H$, is a vector, one of the spatial directions is picked out and this is incompatible with the rotational invariance.

In comparison with Batakis' findings on the possible configurations of the $H$-field (not necessarily derived from a homogeneous potential) in diagonal Bianchi models [6] [5] the cases $\chi(d \rightarrow)$ and $\chi(d \nearrow)$ are recovered [18] if Einstein's equations for the diagonal Bianchi $I V$ and VII models are taken into account (primarily the $R_{12}$ constraint equation in the orthonormal frame which implies a solution which is singular everywhere). For the $\chi(d \rightarrow)$ case one must bear in mind that for $Y_{123}=0$ equation (25) implies $X_{012}=0$. Since we started with a purely time-dependent potential the case $\chi(d \uparrow)$ is only partially recovered. However, in the other two cases the generality is not restricted by assuming a purely time-dependent potential.

Eight independent functions of three spatial variables were found to be required to characterise a general inhomogeneous solutions of the string field equations. This was compared with the number required to specify each homogeneous Bianchi type solution. It was found that the most general homogeneous solutions are of Bianchi types III and $\mathrm{VI}_{h=0,-\frac{1}{2},-2}$, and contain 8 independent constants. This situation contrasts with that for spatially homogeneous vacuum and perfect-fluid universes in general relativity and for degenerate string cosmologies with $\phi \neq 0$ and $H=0$. In these cases, the most general universes are of Bianchi types $\mathrm{VI}_{h}, \mathrm{VII}_{h}, \mathrm{VIII}$, and IX. When $H \neq 0$ we find a change in the relative degrees of generality that is analogous to that found in the case of spatially homogeneous general relativistic universes containing pure magnetic fields.

Acknowledgements The authors would like to thank A. Lahiri and M. Dabrowski for discussions. JDB was supported by a PPARC Senior Fellowship and KEK was supported by the German National Scholarship Foundation.

\section{References}

[1] M. B. Green, J. H. Schwarz, E. Witten Superstring Theory, Vol. I (CUP: Cambridge, 1987); E. S. Fradkin, \& A. A. Tseytlin, Nucl. Phys. B 261, 1 (1985); C. G. Callan, E. J. Martinec \& M. J. Perry, Nucl. Phys. B 262, 593 (1985); C. Lovelace, Nucl. Phys. B 273, 413 
(1985).

[2] E. J. Copeland, A. Lahiri, \& D. Wands, Phys. Rev. D 50, 4868 (1994).

[3] E. J. Copeland, A. Lahiri, \& D. Wands, Phys. Rev. D 51, 1569 (1995)

[4] M. Gasperini, J. Maharana \& G. Veneziano, Phys. Lett. B 272, 277 (1991); J. GarcíaBellido, M. Quirós, Nucl. Phys. B368 463 (1992); J. García-Bellido, M. Quirós, Nucl. Phys. B 385558 (1992); M. Gasperini, \& G. Veneziano, Phys. Lett. B 277, 256 (1992); M. Gasperini, R. Ricci \& G. Veneziano, Phys. Lett. B 319, 438 (1993); M. Gasperini \& R. Ricci, Class. Quantum Grav. 12, 677 (1995). A. A. Saaryan, Astrophysics 38, 164 (1995).

[5] N. A. Batakis \& A. A. Kehagias, Nucl. Phys. B 449, 248 (1995); N. A. Batakis, Phys. Lett. B 353, 450 (1995).

[6] N. A. Batakis, Phys. Lett. B 353, 39 (1995).

[7] L. Bianchi, Mem. Soc. It. della Sc. (dei XL) 11, 267 (1897); Lezioni sulla teoria dei gruppi continui finiti di transformazioni, (Spoerri: Pisa, 1918).

[8] A. H. Taub, Ann. Math. 53, 472 (1951).

[9] R. Kantowski \& R. K. Sachs, J. Math. Phys. 7, 443 (1966); A. S. Kompanyeets \& A .S. Chernov, Sov. Phys. JETP 20, 1303 (1964); C. B. Collins, J. Math. Phys. 18, 2116 (1977). Exact string cosmological solutions have been found for Kantowski-Sachs models by J. D. Barrow and M. Dabrowski, Phys. Rev. D 000000 (1996).

[10] L. P. Hughston \& K. C. Jacobs, Astrophys. J. 160, 147 (1969).

[11] G. F. R. Ellis \& M. A. H. MacCallum, Comm. Math. Phys. 12, 108 (1969) and 19, 31 (1970).

[12] See e.g. R. M. Wald, General Relativity (U. Chicago P.: Chicago, 1984).

[13] M. P. Ryan \& L. C. Shepley, Homogeneous Relativistic Cosmologies, (Princeton U. P.; Princeton, 1975).

[14] M. A. H. MacCallum, in General Relativity: an Einstein Centenary, eds. S. W. Hawking \& W. Israel (CUP: Cambridge, 1979); S. T. C. Siklos, in Relativistic Astrophysics and Cosmology, eds. X. Fustero \& E. Verdaguer, (World Scientific: Singapore, 1984), pp 201-248; S. T. C. Siklos, Class. Q. Grav. 13, 1931 (1996); J. D. Barrow \& D. H. Sonoda, Phys. Rep. 139,1 (1986); J.D. Barrow, in The Physical Universe: The Interface Between Cosmology, Astrophysics and Particle Physics, eds. J. D. Barrow, A.B. Henriques, M.T.V.T. Lago \& M.S. Longair, Springer LNP 383, (Springer: NY,1991), pp1-20.

[15] L. Landau \& E. M. Lifshitz, The Classical Theory of Fields, 4th edn., (Pergamon: Oxford, 1975).

[16] $h=-\frac{1}{9}$ is not the special value because Ryan and Shepley, in ref. [13], use an offdiagonal parametrization for $m_{\alpha \beta}$ in Bianchi $\mathrm{VI}_{h \neq-1} ; h=-\frac{1}{9}$ is obtained as special value if one chooses the diagonal parametrization.

[17] V. A. Ruban, Sov. Astron. 26, 632 (1983) and 29, 5 (1985).

[18] $\chi(d \quad)$ is a parameter with value I,II,...,IX denoting the Bianchi classification, $d$ stands for diagonal and an arrow indicates the orientation of $* H=* H_{0}(t) d t+* H_{\alpha}(t) \omega^{\alpha}$. So that in 
a $\chi(d \uparrow)$ model $* H$ has only a timelike component, in a $\chi(d \rightarrow)$ model only spatial components while in a $\chi(d \nearrow)$ model both components appear. 


\begin{tabular}{|c|c|c|c|c|c|c|c|c|c|}
\hline Bianchi Type & $a_{\alpha}$ & $\mathbf{m}$ & $V_{\alpha}$ & $X^{0 \alpha \beta}$ & $Y_{123}$ & $p$ & $r$ & $u$ & $\overline{\mathcal{N}}$ \\
\hline I & 0 & 0 & $V_{\alpha}$ arb & $\begin{array}{c}X^{012}=e^{2 \phi} K_{3} \\
X^{013}=-e^{2 \phi} K_{2} \\
X^{023}=e^{2 \phi} K_{1}\end{array}$ & 0 & 0 & 3 & 0 & 6 \\
\hline II & 0 & $\operatorname{diag}(1,0,0)$ & $\begin{array}{l}V_{1}=0 \\
V_{2} \text { arb } \\
V_{3} \text { arb }\end{array}$ & $\begin{array}{c}X^{012}=e^{2 \phi} K_{3} \\
X^{013}=-e^{2 \phi} K_{2} \\
X^{023}=0\end{array}$ & 0 & 3 & 1 & 1 & 6 \\
\hline $\mathbf{V I}_{-1}$ & 0 & $\mathbf{m}=-\alpha$ & $\begin{array}{l}V_{1}=0 \\
V_{2}=0 \\
V_{3} \text { arb }\end{array}$ & $\begin{array}{c}X^{012}=e^{2 \phi} K_{3} \\
X^{013}=0 \\
X^{023}=0\end{array}$ & 0 & 5 & 0 & 2 & 6 \\
\hline $\mathbf{V I I}_{0}$ & 0 & $\operatorname{diag}(-1,-1,0)$ & $\begin{array}{l}V_{1}=0 \\
V_{2}=0 \\
V_{3} \text { arb }\end{array}$ & $\begin{array}{c}X^{012}=e^{2 \phi} K_{3} \\
X^{013}=0 \\
X^{023}=0\end{array}$ & 0 & 5 & 0 & 2 & 6 \\
\hline VIII & 0 & $\operatorname{diag}(-1,1,1)$ & $\begin{array}{c}V_{1}=0 \\
V_{2}=0 \\
V_{3}=0\end{array}$ & $\begin{array}{l}X^{012}=0 \\
X^{013}=0 \\
X^{023}=0\end{array}$ & 0 & 6 & 0 & 3 & 6 \\
\hline IX & 0 & $\operatorname{diag}(1,1,1)$ & $\begin{array}{c}V_{1}=0 \\
V_{2}=0 \\
V_{3}=0\end{array}$ & $\begin{aligned} X^{012} & =0 \\
X^{013} & =0 \\
X^{023} & =0\end{aligned}$ & 0 & 6 & 0 & 3 & 6 \\
\hline III & $-\frac{1}{2} \delta_{3}^{\alpha}$ & $-\frac{1}{2} \alpha$ & $\begin{array}{l}V_{1}=0 \\
V_{2} \text { arb } \\
V_{3} \text { arb }\end{array}$ & $\begin{array}{c}X^{012}=e^{2 \phi} K_{3} \\
X^{013}=-e^{2 \phi} K_{2} \\
X^{023}=0\end{array}$ & $-S_{12}$ & 5 & 0 & 0 & 8 \\
\hline IV & $-\delta_{3}^{\alpha}$ & $\operatorname{diag}(1,0,0)$ & $\begin{array}{c}V_{1}=0 \\
V_{2}=0 \\
V_{3} \text { arb }\end{array}$ & $\begin{array}{c}X^{012}=e^{2 \phi} K_{3} \\
X^{013}=0 \\
X^{023}=0\end{array}$ & $-2 S_{12}$ & 5 & 0 & 1 & 7 \\
\hline $\mathbf{V}$ & $-\delta_{3}^{\alpha}$ & 0 & $\begin{array}{c}V_{1}=0 \\
V_{2}=0 \\
V_{3} \text { arb }\end{array}$ & $\begin{array}{c}X^{012}=e^{2 \phi} K_{3} \\
X^{013}=0 \\
X^{023}=0\end{array}$ & $-2 S_{12}$ & 3 & 0 & 1 & 5 \\
\hline $\begin{array}{l}h=0 \\
\mathbf{V} \mathbf{I}_{h} \neq-1 \\
\quad h \neq 0,-\frac{1}{2},-2 \\
\quad h=-\frac{1}{2},-2\end{array}$ & $-\frac{1}{2}(h+1) \delta_{3}^{\alpha}$ & $-\frac{1}{2}(h-1) \alpha$ & $\begin{array}{l}V_{1}=0 \\
V_{2} h=0 \\
V_{3} \text { arb }\end{array}$ & $\begin{array}{c}X^{012}=e^{2 \phi} K_{3} \\
X_{h=0}^{013}=-e^{2 \phi} K_{2} \\
X_{h \neq 0}^{013}=0 \\
X^{023}=0\end{array}$ & $-(h+1) S_{12}$ & $\begin{array}{l}5 \\
5 \\
5\end{array}$ & $\begin{array}{l}0 \\
1\end{array}$ & $\begin{array}{l}1 \\
1\end{array}$ & $\begin{array}{l}7 \\
8\end{array}$ \\
\hline $\mathbf{V I I}_{h \neq 0}$ & $-\frac{h}{2} \delta_{3}^{\alpha}$ & $\begin{array}{c}\operatorname{diag}(-1,-1,0) \\
+\frac{h}{2} \alpha\end{array}$ & $\begin{array}{l}V_{1}=0 \\
V_{2}=0 \\
V_{3} \text { arb }\end{array}$ & $\begin{array}{c}X^{012}=e^{2 \phi} K_{3} \\
X^{013}=0 \\
X^{023}=0\end{array}$ & $-\frac{h}{2} S_{12}$ & 5 & 0 & 1 & 7 \\
\hline
\end{tabular}

Table 1: Summary of the possible components of the homogeneous antisymmetric tensor field strength and degrees of freedom. The different1 jariables are explained in the text. 